\title{
Intermittent fasting decreases oxidative stress parameters in Wistar rats (Rattus norvegicus)
}

\author{
Titis Nurmasitoh*, Shindy Yudha Utami**, Endah Kusumawardani**, \\ Abdulhalim Ahmad Najmuddin**, and Ika Fidianingsih***
}

\begin{abstract}
\section{BACKGROUND}

Chronic and degenerative diseases are closely related to modern lifestyles that tend to be deficient in physical activity but excessive in food intake. One method used to overcome this problem is dietary restriction through intermittent fasting. Intermittent fasting decreases the risk of chronic and degenerative diseases, e.g. by lowering oxidative stress. Oxidative stress can be determined from the malondialdehyde (MDA) levels and lipid profile in the blood. The present study aimed to determine the effect of intermittent fasting on plasma MDA levels and lipid profile of Wistar rats (Rattus norvegicus).
\end{abstract}

\section{METHODS}

This research was a laboratory experimental research using a post-test control group design. Twenty four male Wistar rats (Rattus norvegicus) were randomly assigned to 4 groups, ie control group (K), fasting group (P), non-fasting obese group (OTP), and fasting obese group (OP). Fasting treatment given in this research was intermittent fasting, comprising one day of fasting for 12 hours alternating with one day of normal feed ad libitum in the span of 8 weeks. After completion of treatment, blood was taken transcardially for examination of MDA levels and lipid profiles using spectrophotometry.

\section{RESULTS}

There were significant between-group differences in MDA levels and lipid profiles ( $\mathrm{p}<0.05$ for all parameters). Groups treated with intermittent fasting had lower levels of MDA, total cholesterol, triglycerides, and LDL than those without intermittent fasting. The HDL levels were higher in the intermittent fasting group than the other groups.

\section{CONCLUSION}

Intermittent fasting has an effect on the oxidative stress parameters of Wistar rats (Rattus norvegicus).

Keywords: Intermittent fasting, diet restriction, MDA, lipid profile, oxidative stress, rats
*Department of Physiology,

Faculty of Medicine,

Islamic University of Indonesia

Yogyakarta

**Faculty of Medicine,

Islamic University of Indonesia,

Yogyakarta

****Department of Histology,

Faculty of Medicine,

Islamic University of Indonesia,

Yogyakarta

\section{Correspondence:}

dr. Titis Nurmasitoh, MSc

Department of Physiology,

Faculty of Medicine,

Islamic University of Indonesia,

Yogyakarta

Email: titisnurmasitoh@gmail.com

Date of first submission, June 10, 2017

Date of final revised submission, April 9, 2018

Date of acceptance, April 10, 2018

This open access article is distributed under a Creative Commons AttributionNon Commercial-Share Alike 4.0 International License

Cite this article as: Nurmasitoh T, Utami SY, Kusumawardani E, Najmuddin AA, Fidianingsih I. Intermittent fasting decreases oxidative stress parameters in Wistar rats (Rattus norvegicus). Univ Med 2018;37:31-8. doi: 10.18051/ UnivMed.2018.v37.31-38 


\section{INTRODUCTION}

The lifestyle changes occurring in various community groups also influence their health status. In post-industrial and industrializing countries, including Indonesia, the number of cases with obesity is continually increasing. ${ }^{(1)}$ The prevalence of obesity in Indonesian females increased by $32.9 \%$ from 2007 up to and including 2013. The prevalence of adult male inhabitants with obesity was $19.7 \%$ in 2013 , which was higher than the $13.9 \%$ in 2007.(2) Globally, the proportion of adults with body mass index (BMI) of $25 \mathrm{~kg} / \mathrm{m}^{2}$ increased from 1980 to 2013 , i.e. from $28.8 \%$ to $36.9 \%$ in males and from $29.8 \%$ to $38 \%$ in females. ${ }^{(3)}$

Obesity is closely associated with modern lifestyles that tend to be deficient in physical activity and excessive in food consumption. Changes in dietary pattern also contribute to the cases of obesity. Currently the public tends to consume foods excessive in calories but very deficient in the essential nutrients actually required by the body. ${ }^{(2-4)}$ The cases of obesity are caused by errors in nutritional management and are strongly suspected to increase the risk for degenerative diseases, such as diabetes, arteriosclerosis, hypertension, cardiovascular disease, stroke, heart failure, cancer, etc. ${ }^{(4,5)} \mathrm{A}$ number of studies demonstrated that a variety of diseases caused by obesity are associated with oxidative stress and involve inflammatory mechanisms. ${ }^{(6,7)}$

A number of studies have been conducted to prevent obesity. One of the frequently used methods is dietary restriction. ${ }^{(7,8)}$ Dietary restriction is applied by various methods and in various diseases with sufficiently diverse parameters. Dietary restriction is thought to be associated with a decreased risk for cardiovascular disease, cancer, and a number of other chronic diseases through various mechanisms, one of them being a decrease in oxidative stress. Oxidative stress is determined by measuring malondialdehyde (MDA) concentrations and the lipid profile in the blood. ${ }^{(7,9,10)}$ The methods of dietary restriction of the various studies are sufficiently diverse. For instance, there are studies that restrict the feeding frequency or daily amount of calories in healthy rats, ${ }^{(11,12)}$ and the percentage of intake in rats with induced diabetes mellitus. ${ }^{(10)}$ There are also various models of dietary restriction that are used in given regions or community groups, such as the Okinawan diet, ${ }^{(1)}$ the Ramadan fast, ${ }^{(9)}$ the Greek Orthodox fast, ${ }^{\left({ }^{(9)}\right.}$ and the Daniel fast. ${ }^{(9)}$ Previous studies on intermittent fasting also used different techniques and caloric restrictions from those of the present study. Intermittent fasting as used in the present study, comprising one day of fasting for 12 hours alternating with one day of the normal diet, is still infrequently used. The parameters under study are also diverse, such as superoxide dismutase (SOD), ${ }^{(7)}$ ROS production, ${ }^{(11,13)}$ redox status, ${ }^{(11,13)} \mathrm{MDA},{ }^{(7,11)}$ insulin sensitivity ${ }^{(14)}$ diabetes mellitus, ${ }^{(7)}$ cancer, ${ }^{(15)}$ nutritional status, ${ }^{(16)}$ lipid profile, ${ }^{(10)}$ lifespan and anti-aging, ${ }^{(9,17)}$ memory, ${ }^{(12)}$ and others. The majority of these studies state that dietary restriction has a positive effect on the body. Among these studies, those using dietary restriction by the method of intermittent fasting such as applied in the present study (one day fasting for 12 hours and one day normal diet) with parameters of oxidative stress, i.e. increase in MDA concentrations and lipid profile in rats, have not been carried out. One study using the method of intermittent fasting that is similar to this study was conducted by Fidianingsih et al., ${ }^{(18)}$ but these investigators looked for the effect of intermittent fasting on the histologic structure of various organs associated with the immune system.

The purpose of the present study was to evaluate the effect of intermittent fasting on oxidative stress markers in rats, i.e. the concentrations of MDA, total cholesterol, triglycerides, low-density lipoprotein (LDL), and high-density lipoprotein (HDL). 


\section{METHODS}

\section{Research design}

This was an experimental laboratory study with post-test control group design. The study was conducted at the Integrated Research Laboratory, Faculty of Medicine, Universitas Islam Indonesia (UII) for 6 months, from June to December 2015

\section{Subjects of the study}

This study used 24 male Wistar rats (Rattus norvegicus) aged around 8 weeks and weighing 160-260 grams, which were obtained from the Test Animal Laboratory, Integrated Research and Testing Laboratory (LPPT), Gadjah Mada University (UGM), Yogyakarta. The number of test animals was adjusted using the $3 \mathrm{R}$ principle (replacement, refinement, and reduction), to minimize the use of experimental animals, and was calculated with the following resource equation: ${ }^{(19)} \mathrm{E}=\mathrm{N}-\mathrm{T}$, where $\mathrm{E}=$ constant between $10-20, \mathrm{~N}=$ intended number of animals per group multiplied by the number of intervention groups, while $\mathrm{T}=$ number of intervention groups ( 4 in this study). If the number of animals per group $=$ 6 then $E=(6 \times 4)-4=20$, which is still in the range of 10-20. Therefore, the sample size or total number of test animals is $24 .{ }^{(19)}$

\section{Intervention}

Previous to the intervention, the rats were adapted for 7 days. The test animals were placed in cages in a room with controlled light intensity, using a 12- hour light-dark cycle. During the adaptation period, feed and drinking water were given ad libitum.

In the second week (after completion of the adaptation), the rats were grouped randomly into 4 groups, i.e. control group (K), fasting group (P), non-fasting obese group (OTP), and fasting obese group (OP). Each of the groups consisted of 6 rats that were assigned randomly by lot to place them in the designated groups. Group $\mathrm{K}$ was given standard rat feed and plain drinking water from the onset to the end of the study (12 weeks).
Group $P$ was subjected to intermittent fasting, i.e. one day of fasting for 12 hours and one day of normal diet, from the onset to the end of the study according to the protocol of Fidianingsih et al. ${ }^{(18)}$ Group OTP was the group on high fat feed for 4 weeks followed by 8 weeks of normal feed and plain drinking water until the end of the study. Group OP was the group on high-fat feed for 4 weeks followed by 8 weeks of intermittent fasting until the end of the study. Before undergoing the intervention, all rats were weighed to determine their weight at baseline. The rats were weighed every day to determine changes in body weight from day to day. The weighing was performed every morning using rat scales and the weights recorded. In addition to weighing, the body length of the rats was measured according to the procedure of Novelli et al. ${ }^{(20)}$ The rats were measured in the supine position from the nose to the anus using a ruler. The body length was determined twice, i.e. at the start and end of induction of obesity to determine the body mass index (BMI) of the rats, using the formula: body weight (gram)/length ${ }^{2}\left(\mathrm{~cm}^{2}\right)$.

The fasting of the groups $\mathrm{P}$ and $\mathrm{OP}$ was intermittent fasting, i.e. the rats were fasted for 12 hours from 18.00 to 06.00 , then were not fasted on the second day, again fasted on the third day, and not fasted on the fourth day, etc. On nonfasting days, the rats were given feed and drinking water ad libitum. In group $\mathrm{P}$, the fasting was performed from the start of the intervention, whereas in group OP, the fasting was performed for 8 weeks after the rats received the high-fat feed for 4 weeks. The feeds for the control group and non-fasting groups were given on average at $10 \%$ of body weight per day or around 20 grams/ day (10 grams was given in the morning and 10 grams in the evening). Induction of obesity was performed using high-fat feed, in the form of a feed mixture consisting of rat feed with the addition of shortening ( $1 / 10$ of the feed by weight), $1 / 2$ egg yolk, and 7 grams of white rice until the rats attained obesity, i.e. when the BMI $>0.68$ $\mathrm{g} / \mathrm{cm}^{2}$.(20) 


\section{Laboratory analysis}

At the end of the study, blood was drawn transcardially from each rat using a $3-\mathrm{ml}$ syringe and around $2 \mathrm{ml}$ was placed in an EDTA tube. Subsequently, the blood samples were transported to the Nutritional Laboratory of the Inter-University Center (Pusat Antar Universitas, PAU), UGM, Yogyakarta to determine the concentrations of MDA, total cholesterol, triglycerides, and LDL by enzymatic spectrophotometry. The blood was then centrifuged to obtain the supernatant. To every 100 microliters of serum, $2.45 \mathrm{ml}$ of TCA (trichloracetic acid) and $2.45 \mathrm{ml}$ of TBA (thiobarbituric acid) were added, then the mixture was heated at $100^{\circ} \mathrm{C}$ for 20 minutes. Subsequently, the mixture was centrifuged for 10 minutes at $8000 \mathrm{rpm}$. The absorbance of the obtained supernatant was determined with a blank of $2.45 \mathrm{ml}$ TCA and $2.45 \mathrm{ml}$ TBA based on the included standard curves.

\section{Statistical analysis}

The distribution of the obtained numerical data was determined with the Saphiro-Wilk test, followed by Levene's test to assess the homogeneity of the data. One-way Anova was used to determine between-group differences in the mean concentrations of MDA, total cholesterol, triglycerides, LDL, and HDL. If there was a significant difference, a multiple comparison post-hoc test was done. The difference was considered significant if $\mathrm{p}<0.05$.

\section{Ethical clearance}

The present study was approved by the Ethics Committee, Faculty of Medicine, UII, as stated in letter of ethical clearance No. 18/ Ka.Kom.Et/70/KE/III/2015.

\section{RESULTS}

Before the start of the intervention, the randomly grouped rats were weighed to make sure that they were in the same condition at the start of the study. Mean weights of the rats were analyzed by ANOVA to determine significant differences in body weight before the intervention.

Analysis using one-way Anova showed no significant difference in the weight of the rats between groups at baseline, indicating that the randomization successfully equalized the weights among the four groups.

After the induction of obesity for 4 weeks, the mean BMI of the rats in the induction groups (OTP and OP) was more than 0.68 , whereas the control group showed a mean BMI of less than $0.68 .^{(20)}$ After completion of the induction, the intervention was continued for each group according to schedule until 8 weeks afterward. On the last day, blood was drawn from the heart of each rat to a volume of $2 \mathrm{ml}$ to determine the mean concentrations of MDA, total cholesterol, triglycerides, LDL, and HDL, as shown in Table 1.

The concentrations of MDA, total cholesterol, triglycerides, and LDL at the end of the study were highest in group OTP (the group with induction of obesity without fasting) and lowest in group P (fasting without induction of obesity). On the other hand, the highest HDL concentration was shown by the rats in the fasting group and the lowest concentration by

Table 1. Mean MDA, total cholesterol, triglycerides, LDL, and HDL

\begin{tabular}{lccccc}
\hline \multicolumn{1}{c}{ Group } & $\mathbf{C}(\mathbf{n}=\mathbf{6})$ & $\mathbf{F ~ ( n = 6 )}$ & NFO $(\mathbf{n}=\mathbf{6})$ & $\mathbf{F O}(\mathbf{n}=\mathbf{6})$ & $\mathbf{p}^{*}$ \\
\hline MDA $(\mathrm{nmol} / \mathrm{mL})$ & $2.11 \pm 0.18$ & $1.67 \pm 0.19$ & $03.15 \pm 0.14$ & $2.52 \pm 0.14$ & 0.000 \\
Cholesterol $(\mathrm{mg} / \mathrm{dL})$ & $128.83 \pm 1.26$ & $119.71 \pm 1.48$ & $152.43 \pm 2.50$ & $138.69 \pm 2.69$ & 0.000 \\
Triglycerides $(\mathrm{mg} / \mathrm{dL})$ & $100.58 \pm 2.96$ & $89.93 \pm 3.22$ & $117.94 \pm 2.54$ & $109.61 \pm 2.61$ & 0.000 \\
LDL (mg/dL) & $50.28 \pm 2.22$ & $38.57 \pm 2.22$ & $58.31 \pm 1.91$ & $55.63 \pm 1.66$ & 0.000 \\
HDL (mg/dL) & $48.35 \pm 2.18$ & $56.09 \pm 2.09$ & $36.18 \pm 1.62$ & $42.21 \pm 1.58$ & 0.000 \\
\hline
\end{tabular}

Group C: control group, group F: fasting group, group NFO: non-fasting obese group, group FO: fasting obese group

* Results of one-way Anova 
Table 2. Post hoc LSD analysis

\begin{tabular}{|c|c|c|c|}
\hline \multirow{2}{*}{ MDA } & \multicolumn{2}{|r|}{ Mean difference } & \multirow[t]{2}{*}{ p } \\
\hline & & & \\
\hline \multirow[t]{3}{*}{$\mathrm{C}$} & $\mathrm{F}$ & 0.6433 & 0.000 \\
\hline & NFO & -0.84 & 0.000 \\
\hline & FO & -0.21 & 0.000 \\
\hline \multirow[t]{2}{*}{$\mathrm{F}$} & NFO & -1.285 & 0.000 \\
\hline & FO & -0.655 & 0.000 \\
\hline NFO & FO & 0.8283 & 0.000 \\
\hline \multicolumn{4}{|c|}{ Cholesterol } \\
\hline \multirow[t]{3}{*}{$\mathrm{C}$} & $\mathrm{F}$ & 12.7511 & 0.000 \\
\hline & NFO & -19.9689 & 0.000 \\
\hline & FO & -6.2289 & 0.000 \\
\hline \multirow[t]{2}{*}{$\mathrm{F}$} & NFO & -29.0939 & 0.000 \\
\hline & FO & -15.3539 & 0.000 \\
\hline NFO & FO & 17.3661 & 0.000 \\
\hline \multicolumn{4}{|c|}{ Triglycerides } \\
\hline \multirow[t]{3}{*}{$\mathrm{C}$} & $\mathrm{F}$ & 14.076 & 0.000 \\
\hline & NFO & -13.934 & 0.000 \\
\hline & FO & -5.599 & 0.000 \\
\hline \multirow[t]{2}{*}{$\mathrm{F}$} & NFO & -24.5823 & 0.000 \\
\hline & FO & -16.2473 & 0.000 \\
\hline NFO & FO & 11.7627 & 0.000 \\
\hline \multicolumn{4}{|c|}{ Low-density lipoprotein } \\
\hline \multirow[t]{3}{*}{$\mathrm{C}$} & $\mathrm{F}$ & 14.1375 & 0.000 \\
\hline & NFO & -5.5958 & 0.000 \\
\hline & FO & -2.9225 & 0.000 \\
\hline \multirow[t]{2}{*}{$\mathrm{F}$} & NFO & -17.3042 & 0.000 \\
\hline & FO & -14.6308 & 0.000 \\
\hline NFO & FO & 5.1025 & 0.000 \\
\hline \multicolumn{4}{|c|}{ High-density lipoprotein } \\
\hline \multirow[t]{3}{*}{$\mathrm{C}$} & $\mathrm{F}$ & -5.4663 & 0.000 \\
\hline & NFO & 14.4437 & 0.000 \\
\hline & FO & 8.412 & 0.000 \\
\hline \multirow[t]{2}{*}{$\mathrm{F}$} & NFO & 22.1804 & 0.000 \\
\hline & FO & 16.1487 & 0.000 \\
\hline NFO & $\mathrm{FO}$ & -3.7613 & 0.000 \\
\hline
\end{tabular}

the rats in group OTP (induction of obesity without fasting).

The results of one-way Anova showed that there was a significant difference in the concentrations of MDA, total cholesterol, triglycerides, LDL, and HDL between the groups $(p<0.05)$. The post hoc test using LSD showed a significant difference $(p<0.05)$ between the groups for all parameters (Table $2)$. The concentrations of MDA, cholesterol, triglycerides, LDL, and HDL in the fasting groups differed significantly with those in the non-fasting groups.

\section{DISCUSSION}

Intermittent fasting is one of the dietary restriction methods. Several studies state that dietary restriction may influence health in general, prevent premature aging with all of its consequences, prevent degenerative disease, cognitive deficit, etc. ${ }^{(5,9)}$ This is based on the fact that various diseases, particularly degenerative diseases, are frequently caused by lifestyle, in this case including uncontrolled dietary patterns. ${ }^{(1)}$ In addition, dietary restriction, such as alternateday fasting and calorie restriction may decrease the risk of various chronic diseases, such as diabetes mellitus, heart disease, and cancer, and at the same time prevent their occurrence. ${ }^{(10)}$ Therefore dietary restriction is expected to be able to improve diseases that are the result of uncontrolled lifestyle.

There are various dietary restriction methods, one of them being intermittent fasting, which itself has been developed into various models. ${ }^{(1,9)}$ The method of intermittent fasting was first introduced by Carlson and Hoelzel, who found that rats subjected to intermittent fasting had increased life spans. ${ }^{(11)}$

Intermittent fasting may significantly improve oxidative stress parameters as seen in the lower concentrations of MDA, total cholesterol, triglycerides, and LDL, and the higher concentrations of HDL. In the fasting groups, the oxidative stress parameters showed the most excellent results compared with the other groups. Administration of intermittent fasting to groups with induced obesity also showed significant improvement in oxidative stress parameters, as shown in the decreased concentrations of MDA, total cholesterol, triglycerides, and LDL, and the increased concentrations of HDL compared with the obese groups that were not subjected to fasting.

The results of the present study showed that plasma MDA concentrations in rats subjected to intermittent fasting were lower compared to the groups not subjected to intermittent fasting. This 
study is in line with a previous study carried out by Chausse et al., ${ }^{(1)}$ who found that intermittent fasting decreased the redox status of specific organs, particularly the liver and brain, so preventing oxidative damage in these organs. More specifically, intermittent fasting decreases the MDA concentrations in the hearts of the rats. This shows that oxidative stress in rat hearts may be minimized through intermittent fasting. Malondialdehyde (MDA) is the main product of lipid peroxidation and is used to determine the amount of reactive oxygen species (ROS) produced by oxidative stress. ${ }^{(6)}$ This agrees with the theory on the effect of dietary restriction, including intermittent fasting. Intermittent fasting is frequently associated with the prevention of oxidative stress in the body. It is well known that excessive and unlimited dietary calories promote lipid peroxidation. Polyunsaturated fatty acids are easily oxidized into free radicals and other reactive compounds such as $\mathrm{H}_{2} \mathrm{O}_{2}$. Lipid peroxidation is a mechanism of cellular injury and is used as an indicator of oxidative stress in cells and tissues. ${ }^{(7,11)}$ Lipid endoperoxides originating from polyunsaturated fatty acids are unstable and are decomposed into a number of complex compounds, including reactive carbonyls, particularly MDA. ${ }^{(6)}$ Restriction of calories and control of the amount of calories entering the body are expected to prevent and control oxidative stress in the body. Therefore, the concentrations of MDA formed in the body also become lower. ${ }^{(6,7)}$

The results of the present study also showed lower total cholesterol, triglyceride, and LDL concentrations in rats subjected to intermittent fasting compared with groups not subjected to intermittent fasting. In agreement with the present study, Klempel also state that in humans, intermittent fasting combined with calorie restriction showed effective results in weight reduction in female subjects with obesity. In addition, this method is also effective in decreasing the risk for cardiovascular disease such as coronary heart disease. The decreased risk for cardiovascular disease is indicated by the decreased concentrations of plasma lipids and LDL. ${ }^{(8)}$ In addition, Chen et al. ${ }^{(14)}$ also state that a restriction to $68 \%$ of carbohydrates may decrease the concentrations of LDL and total cholesterol. Similarly with the present study, Park et al. also conducted a study by dividing rats into 3 groups, i.e. the control group, the group on a high fat diet, and the group on a high fat diet followed with caloric restriction. The calorie restriction that was applied was the administration of $60 \%$ of the number of calories in the normal daily feeds. In the group subjected to caloric restriction, there was a decrease in triglyceride and total cholesterol concentrations, improvement of the leptinadiponectin ratio, a decrease in the expression of NO-synthetase, cyclooxygenase-2, NF-E2related factor, and heme oxygenase-1. ${ }^{(21)}$

Increased dietary intake of energy or fat will increase lipogenesis and subsequently increases the formation of free fatty acids. The free fatty acids are mobilized from adipose tissue into the liver, where they bind to glycerol to form triglycerides. The higher the fat consumption, the higher the triglyceride concentration. Induction with high fat feeds causes increased cholesterol synthesis and ultimately cholesterol accumulation in the blood (hypercholesterolemia). If the blood cholesterol concentrations increase, the LDL as cholesterol transporter to the cells throughout the body also increases. Subsequently, the accumulation of cholesterol is followed by free radical activity causing oxidative damage in a number of tissues. In addition, LDL is also a readily oxidized substance. Oxidized LDL may bind to macrophages and subsequently form foam cells, so causing atherosclerotic lesions. Hypercholesterolemia may cause atherosclerosis, two of the complications being cardiac infarction and stroke. Restriction of dietary intake is expected to decrease the concentrations of triglycerides, LDL, as well as total cholesterol. ${ }^{(22-}$ 24)

In this study we also found higher HDL concentrations in the rats subjected to intermittent fasting compared with the groups not subjected to intermittent fasting. Diets high in carbohydrates 
and polyunsaturated fatty acids decrease apolipoprotein A-1, which is the main component of HDL and functions to decrease oxidized LDL concentrations. Intermittent fasting causes decreased intake of calories so that the production of apolipoprotein A-1 may be optimal and subsequently may increase HDL concentrations in the blood. ${ }^{(25,26)}$ The results of the present study are in line with those of Chen et al. ${ }^{(14)}$ who state that dietary restriction for carbohydrates and calories in Wistar rats was able to increase HDL concentrations and improve other lipid profile parameters. The decrease in triglycerides in the groups on intermittent fasting is the result of the decreased regulation of the hepatic lipogenetic system. Restriction of dietary intake may also increase the activity of lipoprotein lipase (LPL) so increasing triglyceride clearance in the blood vessels. Increases in LPL also increase the catabolism of lipoproteins rich in triglycerides, so resulting in the transfer of esters, apoproteins, and phospholipids to form HDL. ${ }^{(14)}$

The study of Ma et al. ${ }^{(27)}$ also found that mice subjected to dietary interventions alternating between high fat diets and normal showed good results in the prevention and therapy of the metabolic syndrome. The longer the periods of the normal diets replacing the high fat diets, the better the results. Ma et al. ${ }^{(27)}$ divided the groups into $5+1$ (five days of high fat diet alternated by one day of normal diet), 5+2 (five days of high fat diet alternated by two days of normal diet), and $5+3$ (five days of high fat diet alternated by three days of normal diet).

In addition to affecting lipid metabolism, calorie restriction also has an influence on the brain, memory, and other systems. Kuhla et al. also state that caloric restriction (by giving only $60 \%$ of the normal daily requirement) in the long term of up to 74 weeks may prevent a decrease in working memory, cognitive function, and spatial memory in mice. Caloric restriction also causes a decrease in locomotor activity, motor coordination, and increased anxiety in rats. ${ }^{(12)}$

Based on the results of this study and of previous studies, there is a need to conduct further studies to determine the formulation of dietary and caloric restriction as well as the most effective method of intermittent fasting for the reduction of oxidative stress. In addition, further explorations should be carried out with regard to the parameters that may be affected by this restriction of intake. The negative effects that may occur in this intervention should also be investigated. The present study still has its limitations, such as the absence of baseline data, although there are control groups for comparison. The clinical implication of the present study is that dietary restriction may be beneficial in cases of excessive obesity.

\section{CONCLUSIONS}

Intermittent fasting may decrease oxidative stress as shown by the decrease in MDA, total cholesterol, triglyceride, and LDL concentrations.

\section{ACKNOWLEDGMENTS}

The authors express their gratitude to the Directorate of Research and Public Service, Universitas Islam Indonesia, for the funding and to Ernadita Budiastuti for technical assistance in this study .

\section{CONFLICT OF INTEREST}

There was no conflict of interest in the present study .

\section{CONTRIBUTORS}

TN, SYU, EK, AAN, and IF contributed equally to the design of the study, drafting of the manuscript, data collection and analysis, and revising the manuscript. All authors read and approved the final manuscript.

\section{REFERENCES}

1. Willcox DC, Willcox BJ, Todoriki H, et al. The Okinawan diet: Health implications of a lowcalorie, nutrient-dense, antioxidant-rich dietary 
pattern low in glycemic load. J Am Coll Nutr. 2009; 28(supp14):S500-16.

2. Balitbangkes. Riset Kesehatan Dasar (Riskesdas 2013). Jakarta: Kementrian Kesehatan RI; 2013.

3. Ng M, Fleming T, Robinson M, et al. Global, the regional and national prevalence of overweight and obesity in children and adults 1980-2013: a systematic analysis. Lancet 2014;384:766-81. doi: 10.1016/S0140-6736(14)60460-8.

4. Jain S, Singh SN. Calorie restriction: an approach towards obesity management. J Nutr Disord Ther 2015;1(S1). doi: 10.4172/2161-0509.1000S1-006.

5. Klenk J, Rapp K, Ulmer H, et al. Changes of body mass index in relation to mortality: results of a cohort of 42,099 adults. PLoS One 2014;9:1-8. https://doi.org/10.1371/journal.pone.0084817.

6. Sharma SL, Chokshi SA, Desai D, et al. Nonenzymatic antioxidants, malondialdehyde, and total antioxidant activity as markers of oxidativestress in arthritis and rhematoid arthritis. J Med Sci 2013;2:57-60.

7. Sabitha K, Venugopal B, Rafi M, et al. Role of antioxidant enzymes in glucose and lipid metabolism in association with obesity and type 2 diabetes. Am J Med Sci Med 2014;2:21-4. doi: 10.12691/ajmsm-2-1-5.

8. Klempel MC, Kroeger CM, Bhutani S, et al. Intermittent fasting combined with calorie restriction is effective for weight loss and cardioprotection in obese women. Nutr J 2012;11:98. doi: 10.1186/1475-2891-11-98.

9. Trepanowski JF, Canale RE, Marshall KE, et al. Impact of caloric and dietary restriction regimens on markers of health and longevity in humans and animals: a summary of available findings. Nutr J 2011;10:107. doi: 10.1186/1475-2891-10-107.

10. Varady KA, Hellerstein MK. Alternate-day fasting and chronic disease prevention: a review of human and animal trials. Am J Clin Nutr 2007;86:713.

11. Chausse B, Vieira-Lara MA, Sanchez AB, et al. Intermittent fasting results in tissue-specific changes in bioenergetics and redox state. PLoS One 2015;10:1-13. DOI: https://doi.org/10.1371/ journal.pone.0120413.

12. Kuhla A, Lange S, Holzmann C, et al. Lifelong caloric restriction increases working memory in mice. PLoS One 2013;8:7. DOI: https://doi.org/ 10.1371/journal.pone.0068778.

13. Walsh ME, Shil Y, Van Remmen H. The effects of dietary restriction on oxidative stress in rodents. Free Radic Biol Med 2014;66:88-99.

14. Chen JH, Ouyang C, Ding Q, et al. A moderate low-carbohydrate low-calorie diet improves lipid profile, insulin sensitivity and adiponectin expression in rats. Nutrients 2015;7:4724-38.
15. Lv M, Zhu X, Wang H, et al. Roles of caloric restriction, ketogenic diet and intermittent fasting during initiation, progression and metastasis of cancer in animal models: a systematic review and meta-analysis. PLoS One 2014;9:1-17. doi: 10.1371/journal.pone.0115147.

16. Johnstone A. Fasting for weight loss: an effective strategy or latest dieting trend? Int J Obes 2015;39:727-33. doi:10.1038/ijo.2014.214.

17. Roth LW, Polotsky AJ. Can we live longer by eating less? A review of caloric restriction and longevity. Maturitas 2012;71:315-9.

18. Fidianingsih I, Nurmasitoh T, Ashari AD, et al. Structural changes to immune organs in rats after intermittent fasting following a high carb and fat diet. J Pharm Nutr Sci 2018;8:6-12.

19. Charan J, Kantharia N. How to calculate sample size in animal studies? J Pharmacol Pharmacother 2013;4:303.

20. Novelli ELB, Diniz YS, Galhardi CM, et al. Anthropometrical parameters and markers of obesity in rats. Lab Anim 2007;41:111-9.

21. Park S, Park N, Valacchi G, et al. Calorie restriction with a high-fat diet effectively attenuated inflammatory response and oxidative stressrelated markers in obese tissues of the high diet fed rats. Med Inflamm 2012;2012:1-11. doi: 10.1155/2012/984643.

22. Alagwu EA, Okwara JE, Nneli RO, et al. Effect of honey intake on serum cholesterol, triglycerides and lipoprotein levels in albino rats and potential benefits on risks of coronary heart disease. Niger J Physiol Sci 2011;26:161-5.

23. Nurmasitoh T, Pramaningtyas MD. Honey improves lipid profile of diet-induced hypercholesterolemic rats. Univ Med 2015;34: 177-86. doi: 10.18051/UnivMed.2016.v35. 177-186.

24. Tomkin GH, Owens D. LDL as a cause of atherosclerosis. Open Atheroscler Thromb J 2012; 5:13-21.

25. Annema W, von Eckardstein A. High-density lipoproteins. Circ J 2013;77:2432-48. DOI: https:/ /doi.org/10.1253/circj.CJ-13-1025.

26. Das B, Mishra T. Role of HDL-C in health and disease. JIACM 2012;13:218-22.

27. Ma Y, Gao M, Liu D. Alternating diet as a preventive and therapeutic intervention for high fat diet-induced metabolic disorder. Sci Rep 2016; 6:26325. doi: 10.1038/srep26325. 